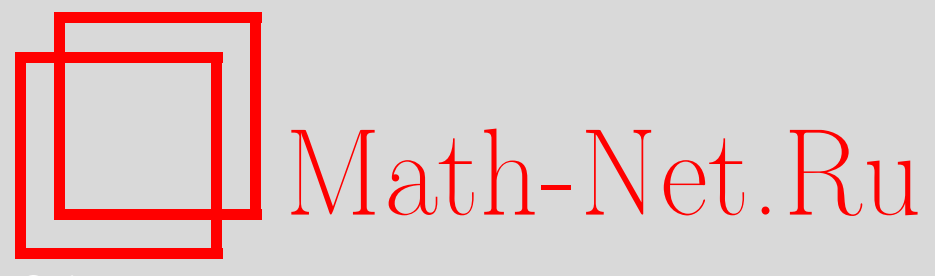

К. Б. Сабитов, И. П. Егорова, О корректности краевых задач с условиями периодичности для уравнения смешанного типа второго рода, Вестн. Сам. гос. техн. ун-та. Сер. Физ.-мат. науки, 2019, номер 3, 430-451

DOI: https://doi.org/10.14498/vsgtu1718

Использование Общероссийского математического портала MathNet.Ru подразумевает, что вы прочитали и согласны с пользовательским соглашением

http: //www . mathnet.ru/rus/agreement

Параметры загрузки:

IP : 54.197 .130 .99

26 апреля 2023 г., 13:44:00

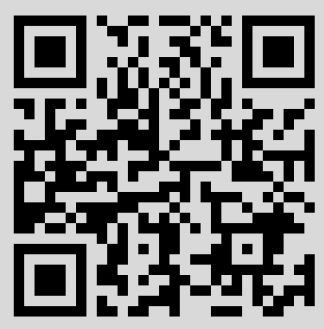


УДК 517.956.6

\title{
О корректности краевых задач с условиями периодичности для уравнения смешанного типа второго рода
}

\author{
К. Б. Сабитов ${ }^{1}$, И. П. Егорова ${ }^{2}$ \\ 1 Стерлитамакский филиал Башкирского государственного университета, \\ факультет математики и информационных технологий, \\ Россия, 453103, Стерлитамак, проспект Ленина, 37. \\ 2 Самарский государственный технический университет, \\ Россия, 443100, Самара, ул. Молодогвардейская, 244.
}

\begin{abstract}
Аннотация
В работе установлены промежутки изменения показателя степени вырождения уравнения смешанного типа с характеристическим вырождением, в которых первая краевая задача и видоизмененная краевая задача (аналог задачи Келдыша) с условиями периодичности поставлены корректно. В случае первой задачи установлен критерий единственности ее решения. Показано, что решение аналога задачи Келдыша единственно с точностью до слагаемого линейной функции. Решения построены в виде суммы ряда по системе собственных функций соответствующей одномерной спектральной задачи. При обосновании сходимости ряда, представляющего решение первой краевой задачи, в классе регулярных решений данного уравнения возникает проблема малых знаменателей более сложной структуры, чем в ранее известных работах, в связи с чем установлена оценка об отделенности от нуля с соответствующей асимптотикой. На основании этой оценки найдены достаточные условия относительно граничных функций для обоснования равномерной сходимости ряда и их производных до второго порядка включительно.
\end{abstract}

Ключевые слова: уравнения смешанного типа, характеристическое вырождение, граничные задачи, условия периодичности, спектральный метод, единственность, малые знаменатели, существование.

Получение: 1 июля 2019 г. / Исправление: 21 августа 2019 г. Принятие: 16 сентября 2019 г. / Публикация онлайн: 11 октября 2019 г.

\section{Научная статья}

(2) (1) Контент публикуется на условиях лицензии Creative Commons Attribution 4.0 International (https://creativecommons.org/licenses/by/4.0/deed.ru)

\section{Образец для цитирования}

Саб и т в К. Б., Е г ор о ва И. П. О корректности краевых задач с условиями периодичности для уравнения смешанного типа второго рода // Вестн. Сам. гос. техн. ун-та. Сер. Физ.-мат. науки, 2019. Т. 23, № 3. С. 430-451. doi: 10.14498/vsgtu1718.

\section{Сведения об авторах}

Камиль Басирович Сабитов (D) https://orcid.org/0000-0001-9516-2704

доктор физико-математических наук, профессор; заведующий кафедрой; каф. математического анализа; e-mail: sabitov_fmf@mail.ru

Ирина Петровна Егорова (1) https://orcid.org/0000-0001-6023-1466

кандидат физико-математических наук, доцент; доцент; каф. высшей математики;

e-mail: ira.egorova81@yandex.ru 


\section{1. Постановка задач. Краткий обзор результатов}

Рассмотрим уравнение смешанного типа второго рода

$$
u_{x x}+(\operatorname{sgn} y)|y|^{s} u_{y y}=0, \quad 0<s<2
$$

в прямоугольной области $D=\{(x, y) \mid 0<x<l,-\alpha<y<\beta\}$. Для уравнения (1) в зависимости от значений параметра $s$ предлагается постановка следующих краевых задач.

ЗАДАча 1. Пусть $0<s \leqslant 1$. Найти в области $D$ функиию $u(x, y)$, удовлетворяюшую следуюшим условиям:

$$
\begin{gathered}
u(x, y) \in C^{1}(\bar{D}) \cap C^{2}\left(D_{+} \cup D_{-}\right) \\
L u \equiv 0, \quad(x, y) \in D_{+} \cup D_{-} ; \\
u(0, y)=u(1, y), \quad u_{x}(0, y)=u_{x}(1, y), \quad-\alpha \leqslant y \leqslant \beta ; \\
u(x, \beta)=f(x), \quad 0 \leqslant x \leqslant l ; \\
u(x,-\alpha)=g(x), \quad 0 \leqslant x \leqslant l,
\end{gathered}
$$

где $f(x), g(x)$ - заданные достаточно гладкие функиии, для которых

$$
f(0)=f(l), \quad g(0)=g(l), \quad f^{\prime}(0)=f^{\prime}(l), \quad g^{\prime}(0)=g^{\prime}(l) .
$$

ЗАДАЧА 2 (АНАЛОГ ЗАДАЧИ КЕЛДЫША) Пусть $1<s<2$. Найmu в области $D$ бункцию $u(x, y)$, удовлетворяющую условиям (2)-(5).

В этой задаче в силу результатов работы М. В. Келдыша [1] в классе функций (2) условие (6) излишне.

Первые исследования для более общего эллиптического уравнения второго порядка от двух переменных с характеристическим вырождением, чем уравнение (1) при $y>0$, провел М. В. Келдыш в работе [1], где он показал, что корректность первой граничной задачи существенным образом зависит от показателя степени вырождения и коэффициента при младшей производной $u_{y}$. На основе этой работы И. Л. Кароль [2] для уравнения (1) в классической области $G$, ограниченной простой жордановой кривой $\Gamma$, лежащей в полуплоскости $y>0$ с концами в точках $O(0,0)$ и $A(1,0)$, характеристиками $O C$ и $A C$ уравнения (1) при $y<0$ поставил задачу Трикоми с данными на кривой $\Gamma$ и характеристике $O C$. В случае, когда контур Г совпадает с «нормальной» кривой, определяемой уравнением

$$
\left(x-\frac{1}{2}\right)^{2}+\left(\frac{2}{2-s}\right)^{2} y^{2-s}=\frac{1}{4}, \quad 0 \leqslant x \leqslant 1,
$$

И. Л. Кароль доказал существование и единственность решения такой задачи при $0<s<1$ методом интегральных уравнений.

Ф. И. Франкль [3] свел задачу определения течения внутри плоскопараллельного симметричного сопла Лаваля заданной формы (прямую задачу теории сопла Лаваля) к новой граничной задаче для уравнения (1) с показателем $s=1 / 2$, которая отличается от задачи Трикоми тем, что на линии изменения типа вместо обычного требования непрерывности производной по нормали к прямой $y=0$ :

$$
u_{y}(x, 0+0)=u_{y}(x, 0-0), \quad 0<x<1,
$$


задается разрывное условие

$$
u_{y}(x, 0+0)=-u_{y}(x, 0-0), \quad 0<x<1 .
$$

В этой же работе он доказал однозначную разрешимость этой задачи при $0<s<1$.

В работе [4] К. Б. Сабитов доказал единственность решения задачи Трикоми и Франкля для уравнения (1) при $0<s<1$ при любой кривой Г из класса Ляпунова методом вспомогательных функций. В следующих работах $[5,6]$ им было показано, что задача Трикоми для уравнения (1) при $s \geqslant 2$ в области $G$ поставлена некорректно. В связи с этим он исследовал задачу Трикоми для уравнения $x^{s} u_{x x}+(\operatorname{sgn} y) u_{y y}=0$ при всех $s>0$.

Новый интерес к задаче Дирихле для уравнений в частных производных возник после известных работ Ф. И. Франкля $[7,8]$, в которых впервые обращено внимание на то, что к этой задаче сводится ряд задач трансзвуковой динамики. Так, например, если рассматривать задачу перехода через звуковой барьер установившихся двумерных безвихревых течений идеального газа в соплах, когда сверзвуковые волны примыкают к стенкам сопла вблизи минимального сечения, то она сводится к задаче Дирихле для уравнений смешанного типа.

В. Б. Шабат [9] исследовал задачу Дирихле для уравнения ЛаврентьеваБицадзе

$$
u_{x x}+(\operatorname{sgn} y) u_{y y}=0
$$

в области $y>-h, 0<h<1 / 2$, гиперболическая часть которой лежит целиком внутри характеристического треугольника $0 \leqslant x+y<x-y \leqslant 1$.

На некорректность задачи Дирихле для уравнения (*) в смешанной области, гиперболическая часть границы которой лежит в характеристическом треугольнике $0 \leqslant x+y<x-y \leqslant 1$, впервые обратил внимание А. В. Бицадзе [10]. Этот факт привлек внимание исследователей к вопросу поиска смешанных областей, для которых задача Дирихле является корректно поставленной.

А. М. Нахушев [11] установил критерий единственности решения задачи Дирихле для уравнений смешанного типа первого рода в цилиндрической области.

В работах А. П. Солдатова $[12,13]$ доказаны теоремы существования и единственности решения задач типа Дирихле для уравнения ЛаврентьеваБицадзе в смешанной области, ограниченной при $y>0$ и $y<0$ соответственно гладкими дугами с общими концами в точках $(0,0)$ и $(0,1)$, при этом дуга при $y<0$ лежит внутри характеристического треугольника.

Следующие авторы методом разделения переменных изучали задачу Дирихле для уравнений смешанного типа в прямоугольной области. В работе J. R. Cannon [14] доказана корректность задачи Дирихле для уравнения Лаврентьева-Бицадзе в прямоугольной области при условии, когда отношение сторон прямоугольника в гиперболической части является натуральным числом.

М. М. Хачев в работах $[15,16]$ исследовал задачу Дирихле для уравнений смешанного типа

$$
(\operatorname{sgn} y)\left[a(x) u_{x x}+b(x) u_{x}+c(x) u\right]+u_{y y}=0
$$




$$
y u_{x x}+u_{y y}=0
$$

и построил решение в виде суммы ряда Фурье.

В работе Р. И. Сохадзе [17] для уравнения

$$
u_{x x}+y u_{y y}+a u_{y}=0, \quad a=\text { const }>0,
$$

исследуется вопрос о существовании хотя бы одного решения задачи Дирихле.

В работе К .Б. Сабитова [18] на основании работы Е. И. Моисеева [19] исследована задача Дирихле для вырождающегося уравнения смешанного типа первого рода

$$
(\operatorname{sgn} y)|y|^{m} u_{x x}+u_{y y}-b^{2}(\operatorname{sgn} y)|y|^{m} u=0, \quad m>0, \quad b \geqslant 0,
$$

в прямоугольной области $D=\{(x, y) \mid 0<x<1,-\alpha<y<\beta\}$, где $\alpha$, $\beta$ - заданные действительные числа. Методом спектрального анализа установлен критерий единственности решения, которое построено в виде суммы ряда Фурье. В этой работе при обосновании сходимости ряда впервые была обнаружена проблема малых знаменателей при обосновании сходимости построенного ряда Фурье, на что предшествующие авторы не обратили внимания при построении решения в виде суммы ряда.

В работе [20] изучена задача Дирихле для уравнения (1) в прямоугольной области $D$. В этой работе при $0<s<1$ установлены критерий единственности и существование решения задачи (2)-(6). Если $1 \leqslant s<2$, то производная $u_{y}(x, y)$ решения уравнения $(1)$, вообще говоря, при $y \rightarrow 0$ обращается в бесконечность. В этом случае задача (2)-(6) переопределена, т. е. для выделения единственности решения аналога этой задачи достаточно задать лишь одно граничное условие на верхнем (5) или нижнем (6) основании прямоугольника $D$.

В работе [21] с использованием метода спектрального анализа [18] изучена краевая задача с условиями периодичности для уравнения (**) в прямоугольной области $D$. Здесь установлен критерий единственности решения. При этом решение задачи построено в виде суммы ряда по системе собственных функций соответствующей одномерной спектральной задачи при всех $m>0$.

В данной работе, следуя [20, 21], установлены классы корректности краевых задач с условиями периодичности для уравнения смешанного типа второго рода (1) в прямоугольной области $D$. В этом состоит существенное отличие от работы [21]. В каждом из этих классов в зависимости от параметра $s$ установлены теоремы единственности и решения задач построены в виде суммы ряда по собственным функциям одномерной спектральной задачи с соответствующим обоснованием сходимости рядов в указанных классах решений данного уравнения. При $0<s \leqslant 1$ установлен критерий единственности решения задачи 1 , т.е. задачи (2)-(6). В случае, когда $1<s<2$, установлен неожиданный новый факт, т.е. доказано, что решение задачи 2 определяется с точностью до слагаемого линейной функции по переменной $y$. 


\section{2. Исследование задачи 1}

2.1. Критерий единственности. Пусть $u(x, y)$-решение задачи $(2)-$ (6). Следуя [18, 20, 21], рассмотрим функции

$$
\begin{gathered}
u_{k}(y)=\sqrt{\frac{2}{l}} \int_{0}^{l} u(x, y) \cos \lambda_{k} x d x, \quad \lambda_{k}=\frac{2 \pi k}{l}, \quad k=1,2, \ldots, \\
u_{0}(y)=\frac{1}{\sqrt{l}} \int_{0}^{l} u(x, y) d x, \\
v_{k}(y)=\sqrt{\frac{2}{l}} \int_{0}^{l} u(x, y) \sin \lambda_{k} x d x .
\end{gathered}
$$

На основании уравнения (1) для функций (7) получим обыкновенное дифференциальное уравнение

$$
u_{k}^{\prime \prime}(y)-(\operatorname{sgn} y)|y|^{-s} \lambda_{k}^{2} u_{k}(y)=0, \quad y \in(-\alpha, 0) \cup(0, \beta) .
$$

В силу того что функция $u(x, y)$ принадлежит классу гладкости $(2)$, для функции $u_{k}(y)$ должны выполняться условия склейки

$$
u_{k}(0+0)=u_{k}(0-0), \quad u_{k}^{\prime}(0+0)=u_{k}^{\prime}(0-0) .
$$

Из граничных условий (5), (6) получим еще два условия для функции $u_{k}(y)$ :

$$
\begin{aligned}
u_{k}(\beta) & =\sqrt{\frac{2}{l}} \int_{0}^{1} u(x, \beta) \cos \lambda_{k} x d x=\sqrt{\frac{2}{l}} \int_{0}^{l} f(x) \cos \lambda_{k} x d x=f_{k}, \\
u_{k}(-\alpha) & =\sqrt{\frac{2}{l}} \int_{0}^{l} u(x,-\alpha) \cos \lambda_{k} x d x=\sqrt{\frac{2}{l}} \int_{0}^{l} g(x) \cos \lambda_{k} x d x=g_{k} .
\end{aligned}
$$

Таким образом, для функций (7) получена краевая задача (10)-(13). В работах $[20,22]$ построено общее решение уравнения (10):

$$
u_{k}(y)= \begin{cases}a_{k} \sqrt{y} I_{\frac{1}{2 q}}\left(p_{k} y^{q}\right)+b_{k} \sqrt{y} K_{\frac{1}{2 q}}\left(p_{k} y^{q}\right), & y>0, \\ c_{k} \sqrt{-y} J_{\frac{1}{2 q}}\left(p_{k}(-y)^{q}\right)+d_{k} \sqrt{-y} Y_{\frac{1}{2 q}}\left(p_{k}(-y)^{q}\right), & y<0,\end{cases}
$$

где $J_{\frac{1}{2 q}}\left(p_{k}(-y)^{q}\right)$ и $Y_{\frac{1}{2 q}}\left(p_{k}(-y)^{q}\right)$ - цилиндрические функции первого и второго рода соответственно; $I_{\frac{1}{2 q}}\left(p_{k} y^{q}\right)$ и $K_{\frac{1}{2 q}}\left(p_{k} y^{q}\right)$ - модифицированные цилиндрические функции; $a_{k}, b_{k}, c_{k}$ и $d_{k}$ - произвольные постоянные; $p_{k}=\lambda_{k} / q$, $q=(2-s) / 2,1 / 2<q<1$ при $0<s<1$.

В (14) постоянные $a_{k}, b_{k}, c_{k}, d_{k}$ подберем так, чтобы выполнялись условия сопряжения (11). Первое из равенств (11) выполнено, если $d_{k}=-\pi b_{k} / 2$ при любых $a_{k}$ и $c_{k}$, а второе равенство - при $c_{k}=\left(\pi b_{k} / 2\right) \operatorname{ctg}(\pi /(4 q))-a_{k}$ и $d_{k}=$ $=-\pi b_{k} / 2$. Тогда функции (14) примут вид

$$
u_{k}(y)= \begin{cases}a_{k} \sqrt{y} I_{\frac{1}{2 q}}\left(p_{k} y^{q}\right)+b_{k} \sqrt{y} K_{\frac{1}{2 q}}\left(p_{k} y^{q}\right), & y \geqslant 0, \\ -a_{k} \sqrt{-y} J_{\frac{1}{2 q}}\left(p_{k}(-y)^{q}\right)+b_{k} \sqrt{-y} \bar{Y}_{\frac{1}{2 q}}\left(p_{k}(-y)^{q}\right), & y \leqslant 0\end{cases}
$$


где

$$
\bar{Y}_{\frac{1}{2 q}}\left(p_{k}\left(-y^{q}\right)\right)=C_{q}\left[J_{\frac{1}{2 q}}\left(p_{k}(-y)^{q}\right)+J_{-\frac{1}{2 q}}\left(p_{k}(-y)^{q}\right)\right], \quad C_{q}=\frac{\pi}{2 \sin (\pi /(2 q))} .
$$

Для нахождения постоянных $a_{k}$ и $b_{k}$ воспользуемся граничными условиями (12) и (13). Удовлетворяя функции (15) граничным условиям (12) и (13), получим систему для нахождения $a_{k}$ и $b_{k}$ :

$$
\left\{\begin{array}{l}
a_{k} I_{\frac{1}{2 q}}\left(p_{k} \beta^{q}\right)+b_{k} K_{\frac{1}{2 q}}\left(p_{k} \beta^{q}\right)=f_{k} \beta^{-\frac{1}{2}} \\
-a_{k} J_{\frac{1}{2 q}}\left(p_{k} \alpha^{q}\right)+b_{k} \bar{Y}_{\frac{1}{2 q}}\left(p_{k} \alpha^{q}\right)=g_{k} \alpha^{-\frac{1}{2}}
\end{array}\right.
$$

Если при всех $k \in \mathbb{N}$ определитель системы (16)

$$
\delta_{k}(\alpha, \beta)=J_{\frac{1}{2 q}}\left(p_{k} \alpha^{q}\right) K_{\frac{1}{2 q}}\left(p_{k} \beta^{q}\right)+\bar{Y}_{\frac{1}{2 q}}\left(p_{k} \alpha^{q}\right) I_{\frac{1}{2 q}}\left(p_{k} \beta^{q}\right) \neq 0,
$$

то она имеет единственное решение:

$$
\begin{aligned}
a_{k} & =\frac{1}{2 \delta_{k}(\alpha, \beta) \sqrt{\alpha \beta}}\left[f_{k} \sqrt{\alpha} \bar{Y}_{\frac{1}{2 q}}\left(p_{k} \alpha^{q}\right)-2 g_{k} \sqrt{\beta} K_{\frac{1}{2 q}}\left(p_{k} \beta^{q}\right)\right], \\
b_{k} & =\frac{1}{2 \delta_{k}(\alpha, \beta) \sqrt{\alpha \beta}}\left[f_{k} \sqrt{\alpha} J_{\frac{1}{2 q}}\left(p_{k} \alpha^{q}\right)-2 g_{k} \sqrt{\beta} I_{\frac{1}{2 q}}\left(p_{k} \beta^{q}\right)\right] .
\end{aligned}
$$

Подставляя найденные значения $a_{k}$ и $b_{k}$, представленные формулами (18) и (19), в формулу (15), найдем окончательный вид функций

$$
u_{k}(y)= \begin{cases}\frac{1}{\delta_{k}(\alpha, \beta)}\left[f_{k} \sqrt{y / \beta} \delta_{k}(\alpha, y)+g_{k} \sqrt{y / \alpha} E_{k}(y, \beta)\right], & y \geqslant 0 \\ \frac{1}{\delta_{k}(\alpha, \beta)}\left[f_{k} \sqrt{-y / \beta} F_{k}(\alpha,-y)+g_{k} \sqrt{-y / \alpha} \delta_{k}(-y, \beta)\right], & y \leqslant 0\end{cases}
$$

где

$$
\begin{gathered}
\delta_{k}(\alpha, y)=J_{\frac{1}{2 q}}\left(p_{k} \alpha^{q}\right) K_{\frac{1}{2 q}}\left(p_{k} y^{q}\right)+\bar{Y}_{\frac{1}{2 q}}\left(p_{k} \alpha^{q}\right) I_{\frac{1}{2 q}}\left(p_{k} y^{q}\right), \\
E_{k}(y, \beta)=I_{\frac{1}{2 q}}\left(p_{k} \beta^{q}\right) K_{\frac{1}{2 q}}\left(p_{k} y^{q}\right)-I_{\frac{1}{2 q}}\left(p_{k} y^{q}\right) K_{\frac{1}{2 q}}\left(p_{k} \beta^{q}\right), \\
F_{k}(\alpha,-y)=\bar{Y}_{\frac{1}{2 q}}\left(p_{k}(-y)^{q}\right) J_{\frac{1}{2 q}}\left(p_{k} \alpha^{q}\right)-\bar{Y}_{\frac{1}{2 q}}\left(p_{k} \alpha^{q}\right) J_{\frac{1}{2 q}}\left(p_{k}(-y)^{q}\right), \\
\delta_{k}(-y, \beta)=J_{\frac{1}{2 q}}\left(p_{k}(-y)^{q}\right) K_{\frac{1}{2 q}}\left(p_{k} \beta^{q}\right)+\bar{Y}_{\frac{1}{2 q}}\left(p_{k}(-y)^{q}\right) I_{\frac{1}{2 q}}\left(p_{k} \beta^{q}\right) .
\end{gathered}
$$

Аналогично получим краевую задачу для функции $v_{k}(y)$ :

$$
\begin{gathered}
v_{k}^{\prime \prime}(y)-(\operatorname{sgn} y)|y|^{m} \lambda_{k}^{2} v_{k}(y)=0, \quad y \in(-\alpha, 0) \cup(0, \beta), \\
v_{k}(0+0)=v_{k}(0-0), \quad v_{k}^{\prime}(0+0)=v_{k}^{\prime}(0-0), \quad k \in \mathbb{N}, \\
v_{k}(\beta)=\sqrt{\frac{2}{l}} \int_{0}^{l} u(x, \beta) \sin \lambda_{k} x d x=\sqrt{\frac{2}{l}} \int_{0}^{l} f(x) \sin \lambda_{k} x d x=\widetilde{f}_{k},
\end{gathered}
$$




$$
v_{k}(-\alpha)=\sqrt{\frac{2}{l}} \int_{0}^{l} u(x,-\alpha) \sin \lambda_{k} x d x=\sqrt{\frac{2}{l}} \int_{0}^{l} g(x) \sin \lambda_{k} x d x=\widetilde{g}_{k} .
$$

Однозначное решение задачи (25)-(28) определяется по формуле

$$
v_{k}(y)= \begin{cases}\frac{1}{\delta_{k}(\alpha, \beta)}\left[\tilde{f}_{k} \sqrt{y / \beta} \delta_{k}(\alpha, y)+\widetilde{g}_{k} \sqrt{y / \alpha} E_{k}(y, \beta)\right], & y \geqslant 0, \\ \frac{1}{\delta_{k}(\alpha, \beta)}\left[\tilde{f}_{k} \sqrt{-y / \beta} F_{k}(\alpha,-y)+\widetilde{g}_{k} \sqrt{-y / \alpha} \delta_{k}(-y, \beta)\right], & y \leqslant 0\end{cases}
$$

где $\delta_{k}(\alpha, y), E_{k}(y, \beta), F_{k}(\alpha,-y), \delta_{k}(-y, \beta)$ определяются соответственно по формулам (21)-(24).

Найдем теперь функцию $u_{0}(y)$. Дифференцируя равенство (8) дважды, учитывая уравнение (1) и граничные условия (4), получим, что функция $u_{0}(y)$ является решением следующей задачи:

$$
\begin{gathered}
u^{\prime \prime}{ }_{0}(y)=0, \quad y \in(-\alpha, 0) \cup(0, \beta), \\
u_{0}(0+0)=u_{0}(0-0), \quad u_{0}^{\prime}(0+0)=u_{0}^{\prime}(0-0), \\
u_{0}(\beta)=\frac{1}{\sqrt{l}} \int_{0}^{l} u(x, \beta) d x=\frac{1}{\sqrt{l}} \int_{0}^{l} f(x) d x=f_{0}, \\
u_{0}(-\alpha)=\frac{1}{\sqrt{l}} \int_{0}^{l} u(x,-\alpha) d x=\frac{1}{\sqrt{l}} \int_{0}^{l} g(x) d x=g_{0} .
\end{gathered}
$$

Единственное решение задачи (30)-(33) имеет вид

$$
u_{0}(y)=\frac{f_{0}-g_{0}}{\alpha+\beta} y+\frac{\alpha f_{0}+\beta g_{0}}{\alpha+\beta}, \quad-\alpha \leqslant y \leqslant \beta .
$$

Поскольку нами найдены явные формулы для функций (7)-(9), на основании полноты тригонометрической системы

$$
\left\{\frac{1}{\sqrt{l}}, \sqrt{\frac{2}{l}} \cos \frac{2 \pi k x}{l}, \sqrt{\frac{2}{l}} \sin \frac{2 \pi k x}{l}\right\}
$$

в пространстве $L_{2}[0, l]$ мы можем доказать единственность решения задачи (2)-(6). Действительно, пусть выполнены условия (17) при всех $n \in \mathbb{N}$ и $f(x)=g(x)=0$. Тогда из формул $(20),(29)$ и $(34)$ следует, что $u_{k}(y) \equiv 0$, $u_{0}(y) \equiv 0, v_{k}(y) \equiv 0$ для $k \in \mathbb{N}$ на $[-\alpha, \beta]$.

Тогда из (7)-(9) имеем

$$
\int_{0}^{l} u(x, y) \cos \lambda_{k} x d x=0, \quad \int_{0}^{l} u(x, y) d x=0, \quad \int_{0}^{l} u(x, y) \sin \lambda_{k} x d x=0 .
$$

Отсюда в силу полноты системы функций (35) в пространстве $L_{2}[0, l]$ следует, что $u(x, y)=0$ почти для всех $x \in[0, l]$ и при любом $y \in[-\alpha, \beta]$. В силу (2) функция $u(x, y) \in C(\bar{D})$, следовательно, $u(x, y) \equiv 0$ в $\bar{D}$. 
Пусть при некотором $k=n \in \mathbb{N}$ нарушено условие $(17)$, т. е. $\delta_{n}(\alpha, \beta)=0$. Тогда однородная задача $(2)-(6)($ где $f(x)=g(x) \equiv 0)$ имеет нетривиальное решение:

$$
u_{n}(x, y)= \begin{cases}\frac{\delta_{n}(\alpha, y) \sqrt{y}}{J_{\frac{1}{2 q}}\left(p_{n} \alpha^{q}\right)} X_{n}(x), & y \geqslant 0 \\ \frac{\delta_{n}(-y, \beta) \sqrt{-y}}{I_{\frac{1}{2 q}}\left(p_{n} \beta^{q}\right)} X_{n}(x), & y \leqslant 0\end{cases}
$$

где $X_{n}(x)=\alpha_{1}+\alpha_{2} \cos \left(\lambda_{n} x\right)+\alpha_{3} \sin \left(\lambda_{n} x\right), \alpha_{i}$ - произвольные постоянные, $i=1,2,3$.

Теперь возникает вопрос о существовании нулей выражения $\delta_{k}(\alpha, \beta)$. Для этого представим его в виде

$$
\delta_{k}(\alpha, \beta)=I_{\frac{1}{2 q}}\left(p_{k} \beta^{q}\right) \gamma_{k}(\alpha, \beta)
$$

где

$$
\gamma_{k}(\alpha, \beta)=J_{\frac{1}{2 q}}\left(p_{k} \alpha^{q}\right) \frac{K_{\frac{1}{2 q}}\left(p_{k} \beta^{q}\right)}{I_{\frac{1}{2 q}}\left(p_{k} \beta^{q}\right)}+\bar{Y}_{\frac{1}{2 q}}\left(p_{k} \alpha^{q}\right)
$$

Поскольку при больших $k$ и любом $\beta>0$ выражение

$$
\frac{K_{\frac{1}{2 q}}\left(p_{k} \beta^{q}\right)}{I_{\frac{1}{2 q}}\left(p_{k} \beta^{q}\right)}=O\left(e^{-2 p_{k} \beta^{q}}\right)
$$

т.е. первое слагаемое из правой части последнего выражения является бесконечно малым более высокого порядка по сравнению со вторым слагаемым, то нули $\gamma_{k}(\alpha, \beta)$ при больших $k$ на малую величину будут отличаться от нулей $\bar{Y}_{\frac{1}{2 q}}\left(p_{k} \alpha^{q}\right)$. Существование нулей функции $\bar{Y}_{\frac{1}{2 q}}\left(p_{k} z\right)$, где $z=\alpha^{q}$, следует из того факта, что функции $\bar{Y}_{\frac{1}{2 q}}\left(p_{k} z\right)$ и $J_{\frac{1}{2 q}}\left(p_{k} z\right)$ являются линейно независимыми решениями уравнения Бесселя

$$
z \frac{d}{d z}\left(z \frac{d}{d z} y(z)\right)+\left(p_{k}^{2} z^{2}-\left(\frac{1}{2 q}\right)^{2}\right) y(z)=0 .
$$

Из общей теории линейных дифференциальных уравнений известно, что нули двух линейно независимых решений уравнения (38) строго чередуются, т. е. на интервале между любыми последовательными нулями любого из этих решений содержится ровно один нуль другого решения. Функция $J_{\frac{1}{2 q}}\left(p_{k} z\right)$ имеет счетное множество положительных нулей. Тогда функция $\bar{Y}_{\frac{1}{2 q}}\left(p_{k} z\right)$ также имеет счетное множество положительных нулей относительно $z=\alpha^{q}$. Следовательно, $\gamma_{k}(\alpha, \beta)$ имеет счетное множество нулей относительно $\alpha^{q}$ независимо от $\beta>0$.

Таким образом, нами установлен следующий критерий единственности решения задачи (2)-(6).

Теорема 1. Если существует решение $и(x, y)$ задачи (2)-(6), то оно единственно тогда и только тогда, когда $\delta_{k}(\alpha, \beta) \neq 0$ при всех $k \in \mathbb{N}$. 
2.2. Существование решения задачи 1. Поскольку $\alpha^{q}$-любое положительное число, оно может принимать значения, близкие к нулям $\gamma_{k}(\alpha, \beta)$. Поэтому при больших $k$ выражение $\gamma_{k}(\alpha, \beta)$ может стать достаточно малым, т. е. возникает проблема малых знаменателей более сложной структуры, чем в известных работах [20, 23].

ЛЕмма 1. Если выполнено одно из следующих условий:

1) $\alpha_{q l}=\alpha^{q} /(q l)$ - любое натуральное число;

2) $\alpha_{q l}=n / m-$ любое дробное число, где $n$ и $m$ - взаимно простые натуральные числа и $m \neq 4$,

то существуют постоянные $C_{0}>0, k_{0}>0\left(k_{0} \in \mathbb{N}\right)$ такие, что при всех $k>k_{0}$ справедлива оценка

$$
\left|\sqrt{k} \gamma_{k}(\alpha, \beta)\right| \geqslant C_{0}>0
$$

Доказ ательст в о. В правой части равенства (37) величина

$$
J_{\frac{1}{2 q}}\left(p_{k} \alpha^{q}\right) \frac{K_{\frac{1}{2 q}}\left(p_{k} \beta^{q}\right)}{I_{\frac{1}{2 q}}\left(p_{k} \beta^{q}\right)}
$$

есть бесконечно малая более высокого порядка, чем $\bar{Y}_{\frac{1}{2 q}}\left(p_{k} \alpha^{q}\right)$ при больших $k$, поэтому достаточно рассмотреть слагаемое

$$
\widetilde{\gamma}_{k}\left(\alpha_{q l}\right)=J_{\frac{1}{2 q}}\left(2 \pi k \alpha_{q l}\right)+J_{-\frac{1}{2 q l}}\left(2 \pi k \alpha_{q l}\right), \quad \alpha_{q l}=\frac{\alpha^{q}}{q l} .
$$

В силу асимптотической формулы функции $J_{\nu}(z)[24, \S 7.13 .1]$ при $z \rightarrow \infty$ :

$$
J_{\nu}(z)=\sqrt{\frac{2}{\pi z}} \cos \left(z-\frac{\pi}{2} \nu-\frac{\pi}{4}\right)+O\left(z^{-\frac{3}{2}}\right)
$$

существует достаточно большое натуральное число $k_{0}$ такое, что при всех $k>k_{0}$ имеем

$$
\sqrt{k} \widetilde{\gamma}_{k}\left(\alpha_{q l}\right)=\frac{2}{\pi \sqrt{\alpha_{q l}}} \cos \left(\frac{\pi}{4 q}\right) \cos \left(2 \pi k \alpha_{q l}-\frac{\pi}{4}\right)=A \cos \left(2 \pi k \alpha_{q l}-\frac{\pi}{4}\right) .
$$

Отсюда видно что, если $\alpha_{q l}=\mu \in \mathbb{N}$, т. е. $\alpha=(\mu q)^{\frac{1}{q}}$, то при $k>k_{0}$

$$
\left|\sqrt{k} \widetilde{\gamma}_{k}\left(\alpha_{q l}\right)\right|=A\left|\cos \left(2 \pi k \mu-\frac{\pi}{4}\right)\right|=A\left|\cos \frac{\pi}{4}\right| \geqslant C_{1}=\text { const }>0 .
$$

Пусть теперь $\alpha_{q l}=n / m-$ рациональное число, где $n$ и $m-$ взаимно простые числа, $m \geqslant 2$. Тогда

$$
\left|\sqrt{k} \widetilde{\gamma}_{k}\left(\alpha_{q l}\right)\right|=A\left|\cos \left(2 \pi k \frac{n}{m}-\frac{\pi}{4}\right)\right|
$$


Разделим $2 k n$ на $m$ с остатком: $2 k n=s m+r, s, r \in \mathbb{N} \cup\{0\}, 0 \leqslant r<m$. Если $r=0$, то этот случай сводится к рассмотренному выше. Пусть $r>0$, тогда последнее выражение примет вид

$$
\left|\sqrt{k} \widetilde{\gamma}_{k}\left(\alpha_{q l}\right)\right|=A\left|\cos \pi\left(\frac{r}{m}-\frac{1}{4}\right)\right|
$$

Поскольку $1 \leqslant r \leqslant m-1$, следовательно

$$
\frac{1}{m}-\frac{1}{4} \leqslant \frac{r}{m}-\frac{1}{4} \leqslant \frac{3}{4}-\frac{1}{m}
$$

Тогда при всех $m \geqslant 2$ имеем

$$
-\frac{1}{4}<\frac{1}{m}-\frac{1}{4} \leqslant \frac{1}{4}, \quad \frac{1}{4} \leqslant \frac{3}{4}-\frac{1}{m}<\frac{3}{4} .
$$

Из оценок (42) видно, что выражение $r / m-1 / 4$ может быть равно $1 / 2$ только при $m=4$ и $r=3$. По условию $m \neq 4$, тогда из (41) следует, что существует положительная постоянная $C_{2}$ такая, что

$$
\left|\sqrt{k} \widetilde{\gamma}_{k}\left(\alpha_{q l}\right)\right| \geqslant C_{2}>0
$$

Далее из неравенств (40) и (43) следует оценка (39) при $k>k_{0}$, где $C_{0}=$ $=\min \left\{C_{1}, C_{2}\right\}$.

Если $\delta_{k}(\alpha, \beta) \neq 0$ при $k=\overline{1, k_{0}}$ и выполнена оценка $(39)$ при $k>k_{0}$, то решение задачи (2)-(6) можно представить в виде суммы ряда Фурье

$$
u(x, y)=\frac{1}{\sqrt{l}} u_{0}(y)+\sqrt{\frac{2}{l}} \sum_{k=1}^{+\infty}\left[u_{k}(y) \cos \lambda_{k} x+v_{k}(y) \sin \lambda_{k} x\right] .
$$

Покажем, что при определенных условиях относительно функций $f(x)$ и $g(x)$ ряд (44) и ряды, полученные из него путем почленного дифференцирования по $x$ и $y$ первого порядка, равномерно сходятся на замкнутой области $\bar{D}$ и дважды почленно дифференцируются по $x$ и $y$ в замкнутой области $\bar{D}_{\varepsilon}=\bar{D} \cap\{|y| \geqslant \varepsilon>0\}$, где $\varepsilon-$ достаточно малое число.

Рассмотрим следующие соотношения:

$$
\begin{array}{lll}
A_{k}(y)=\sqrt{\frac{y}{\beta} \frac{\delta_{k}(\alpha, y)}{\delta_{k}(\alpha, \beta)},} & B_{k}(y)=\sqrt{\frac{y}{\alpha} \frac{E_{k}(\alpha, y)}{\delta_{k}(\alpha, \beta)},} & y \in[0, \beta], \\
C_{k}(y)=\sqrt{\frac{-y}{\beta} \frac{F_{k}(\alpha,-y)}{\delta_{k}(\alpha, \beta)},} & D_{k}(y)=\sqrt{\frac{-y}{\alpha} \frac{\delta_{k}(-y, \beta)}{\delta_{k}(\alpha, \beta)}}, \quad y \in[-\alpha, 0] .
\end{array}
$$

Лемма 2. Пусть выполнена оценка (39) при $k>k_{0}$. Тогда для всех $k>k_{0}$ справедливы следующие оценки:

$$
\begin{gathered}
\left|A_{k}(y)\right| \leqslant C_{3}, \quad\left|A_{k}^{\prime}(y)\right| \leqslant C_{4} k, \quad\left|B_{k}(y)\right| \leqslant C_{5}, \quad\left|B_{k}^{\prime}(y)\right| \leqslant C_{6} k^{1+\lambda}, \quad 0 \leqslant y \leqslant \beta ; \\
\left|A_{k}^{\prime \prime}(y)\right| \leqslant C_{7} k^{2}, \quad\left|B_{k}^{\prime \prime}(y)\right| \leqslant C_{8} k^{2}, \quad \varepsilon \leqslant y \leqslant \beta ;
\end{gathered}
$$




$$
\begin{gathered}
\left|C_{k}(y)\right| \leqslant C_{9} k^{1-\lambda} e^{-k d}, \quad\left|C^{\prime}{ }_{k}(y)\right| \leqslant C_{10} k^{1+\lambda} e^{-k d}, \\
\left|D_{k}(y)\right| \leqslant C_{11}, \quad\left|D^{\prime}{ }_{k}(y)\right| \leqslant C_{12} k^{1+\lambda}, \quad-\alpha \leqslant y \leqslant 0 ; \\
\left|C_{k}^{\prime \prime}(y)\right| \leqslant C_{13} k^{2} e^{-k d}, \quad\left|D_{k}^{\prime \prime}(y)\right| \leqslant C_{14} k^{2}, \quad-\alpha \leqslant y \leqslant-\varepsilon,
\end{gathered}
$$

где $C_{i}-$ здесъ и далее положительные постоянные, $\lambda=1 / 2 q-1 / 2, d=$ $=2 \pi \beta^{q} /(q l)$.

До каз а mель ст в о. Используя асимптотические формулы для цилиндрических функций $[24, \S \S 7.2 .8,7.13 .1]$ в нуле и на бесконечности:

$$
\begin{gathered}
J_{\nu}(z) \sim \frac{1}{\Gamma(1+\nu)}\left(\frac{z}{2}\right)^{\nu}, \quad I_{\nu}(z) \sim \frac{1}{\Gamma(1+\nu)}\left(\frac{z}{2}\right)^{\nu}, \\
K_{\nu}(z) \sim \frac{\Gamma|\nu|}{2}\left(\frac{z}{2}\right)^{-|\nu|}, \quad \nu \neq 0, \quad \text { при } \quad z \rightarrow 0 ; \\
I_{\nu}(z) \sim\left(\frac{1}{2 \pi z}\right)^{1 / 2} e^{z}, \quad J_{\nu}(z) \sim\left(\frac{2}{\pi z}\right)^{1 / 2} \cos \left(z-\frac{\nu \pi}{2}-\frac{\pi}{4}\right), \\
K_{\nu}(z) \sim\left(\frac{\pi}{2 z}\right)^{1 / 2} e^{-z}, \quad \bar{Y}_{\nu}(z) \sim\left(\frac{2}{\pi z}\right)^{1 / 2} \frac{\cos (z-\pi / 4)}{\sin (\nu \pi / 2)} \quad \text { при } \quad z \rightarrow \infty,
\end{gathered}
$$

на основании (21) с учетом (36), (39) при $y \in[0, \beta]$ и достаточно больших $k$ оценим

$$
\left|A_{k}(y)\right| \leqslant \widetilde{C}_{1}\left|\sqrt{k} \bar{Y}_{\frac{1}{2 q}}\left(p_{k} \alpha^{q}\right)\right| \leqslant C_{3}
$$

где $\widetilde{C}_{i}$ - здесь и далее положительные постоянные.

На основании формул дифференцирования цилиндрических функций [24, $\S 7.13 .1]$

$$
\frac{d}{d z}\left[z^{\nu} I_{\nu}(z)\right]=z^{\nu} I_{\nu-1}(z), \quad \frac{d}{d z}\left[z^{\nu} K_{\nu}(z)\right]=-z^{\nu} K_{\nu-1}(z)
$$

найдем

$$
A_{k}^{\prime}(y)=\frac{p_{k} q y^{q-1 / 2}}{\delta_{k}(\alpha, \beta)}\left(-J_{\frac{1}{2 q}}\left(p_{k} \alpha^{q}\right) K_{\frac{1}{2 q}-1}\left(p_{k} y^{q}\right)+\bar{Y}_{\frac{1}{2 q}}\left(p_{k} \alpha^{q}\right) I_{\frac{1}{2 q}-1}\left(p_{k} y^{q}\right)\right) .
$$

Отсюда при $y \in[0, \beta]$ и больших $k$ имеем

$$
\left|A_{k}^{\prime}(y)\right| \leqslant k \widetilde{C}_{2}\left|\sqrt{k} \bar{Y}_{\frac{1}{2 q}}\left(p_{k} \alpha^{q}\right)\right| \leqslant C_{4} k .
$$

Нетрудно показать, что для $A_{k}(y)$ имеет место представление

$$
\left|A_{k}^{\prime \prime}(y)\right|=\left(p_{k} q\right)^{2} y^{2 q-2} A_{k}(y) .
$$

Тогда с учетом оценки (48) из равенства (50) имеем $\left|A_{k}^{\prime \prime}(y)\right| \leqslant \widetilde{C}_{10} k^{2}$.

Аналогично на основании формул (22), (36), (39) и асимптотических формул (46) и (47) оценим функцию $B_{k}(y)$. При $\varepsilon \leqslant y \leqslant \beta$ и больших $k$ имеем:

$$
\left|B_{k}(y)\right| \leqslant\left|\frac{\sqrt{k} \sqrt{y} I_{\frac{1}{2 q}}\left(p_{k} \beta^{q}\right) K_{\frac{1}{2 q}}\left(p_{k} y^{q}\right)}{I_{\frac{1}{2 q}}\left(p_{k} \beta^{q}\right) C_{0}}\right|+\left|\frac{\sqrt{k} \sqrt{y} K_{\frac{1}{2 q}}\left(p_{k} \beta^{q}\right) I_{\frac{1}{2 q}}\left(p_{k} y^{q}\right)}{I_{\frac{1}{2 q}}\left(p_{k} \beta^{q}\right) C_{0}}\right| \leqslant \widetilde{C}_{3} .
$$


Если $0 \leqslant y<\varepsilon$, то аналогично получим

$$
\left|B_{k}(y)\right| \leqslant \widetilde{C}_{4} \sqrt{k}\left|\sqrt{y} K_{\frac{1}{2 q}}\left(p_{k} y^{q}\right)\right| \leqslant \widetilde{C}_{5} k^{-\lambda} .
$$

Из оценок (51) и (52) следует, что $\left|B_{k}(y)\right| \leqslant C_{5}$ при любом $y \in[0, \beta]$.

Используя формулы (49) найдем

$$
B_{k}^{\prime}(y)=\frac{-p_{k} q y^{q-1 / 2}}{\delta_{k}(\alpha, \beta)}\left(I_{\frac{1}{2 q}}\left(p_{k} \beta^{q}\right) K_{\frac{1}{2 q}-1}\left(p_{k} y^{q}\right)+K_{\frac{1}{2 q}}\left(p_{k} \beta^{q}\right) I_{\frac{1}{2 q}-1}\left(p_{k} y^{q}\right)\right)
$$

Тогда отсюда при любом $y \in[0, \beta]$ и больших $k$ имеем

$$
\left|B_{k}^{\prime}(y)\right| \leqslant \widetilde{C}_{6} \sqrt{k}\left|p_{k} q y^{q-1 / 2} K_{\frac{1}{2 q}-1}\left(p_{k} y^{q}\right)\right| \leqslant C_{9} k^{1+\lambda} .
$$

Для функции $B_{k}(y)$ справедливо равенство $B_{k}^{\prime \prime}(y)=\left(p_{k} q\right)^{2} y^{2 q-2} B_{k}(y)$. Отсюда с учетом оценки (51) при $\varepsilon \leqslant y \leqslant \beta$ получим $B_{k}^{\prime \prime}(y) \leqslant C_{8} k^{2}$.

Теперь оценим функции $C_{k}(y)$ и $D_{k}(y)$ при $y \in[-\alpha, 0]$ и достаточно больших $k$. На основании формул $(23),(24),(36),(39)$ и асимптотических формул (46) при $-\varepsilon<y \leqslant 0$ получим следующие оценки для функций $C_{k}(y)$ и $D_{k}(y)$ :

$$
\begin{aligned}
\left|C_{k}(y)\right| \leqslant\left|\frac{\sqrt{k} \sqrt{-y} \bar{Y}_{\frac{1}{2 q}}\left(p_{k}(-y)^{q}\right) J_{\frac{1}{2 q}}\left(p_{k} \alpha^{q}\right)}{I_{\frac{1}{2 q}}\left(p_{k} \beta^{q}\right) C_{0}}\right|+ \\
+\left|\frac{\sqrt{k} \sqrt{-y} \bar{Y}_{\frac{1}{2 q}}\left(p_{k} \alpha^{q}\right) J_{\frac{1}{2 q}}\left(p_{k}(-y)^{q}\right)}{I_{\frac{1}{2 q}}\left(p_{k} \beta^{q}\right) C_{0}}\right| \leqslant C k^{1-\lambda} e^{-k d},
\end{aligned}
$$

$$
\begin{array}{r}
\left|D_{k}(y)\right|=\left|\frac{\sqrt{k} \sqrt{-y} J_{\frac{1}{2 q}}\left(p_{k}(-y)^{q}\right) K_{\frac{1}{2 q}}\left(p_{k} \beta^{q}\right)}{I_{\frac{1}{2 q}}\left(p_{k} \beta^{q}\right) C_{0}}\right|+ \\
+\left|\frac{\sqrt{k} \sqrt{-y} \bar{Y}_{\frac{1}{2 q}}\left(p_{k}(-y)^{q}\right) I_{\frac{1}{2 q}}\left(p_{k} \beta^{q}\right)}{I_{\frac{1}{2 q}}\left(p_{k} \beta^{q}\right) C_{0}}\right| \leqslant C_{11},
\end{array}
$$

так как при любом $y \in[-\alpha, 0]$ и больших $k$

$$
\left|\sqrt{-y} \bar{Y}_{\frac{1}{2 q}}\left(p_{k}(-y)^{q}\right)\right| \leqslant \widetilde{C}_{7} k^{-\frac{1}{2}} \text {. }
$$

Используя формулы сокращенного дифференцирования [24, §7.13.1]

$$
\frac{d}{d z}\left[z^{\nu} J_{\nu}(z)\right]=z^{\nu} J_{\nu-1}(z), \quad \frac{d}{d z}\left[z^{\nu} J_{-\nu}(z)\right]=-z^{\nu} J_{1-\nu}(z),
$$

вычислим

$$
C_{k}^{\prime}(y)=\frac{C_{q} p_{k} q(-y)^{q-1 / 2}}{\delta_{k}(\alpha, \beta)}\left[J_{\frac{1}{2 q}}\left(p_{k} \alpha^{q}\right) J_{1-\frac{1}{2 q}}\left(p_{k}(-y)^{q}\right)+\right.
$$




$$
\left.+J_{-\frac{1}{2 q}}\left(p_{k} \alpha^{q}\right) J_{\frac{1}{2 q}-1}\left(p_{k}(-y)^{q}\right)\right]
$$

$$
\begin{array}{r}
D_{k}^{\prime}(y)=-\frac{p_{k} q(-y)^{q-1 / 2}}{\delta_{k}(\alpha, \beta)}\left[C_{q} I_{\frac{1}{2 q}}\left(p_{k} \beta^{q}\right)\left[J_{\frac{1}{2 q}-1}\left(p_{k}(-y)^{q}\right)-J_{1-\frac{1}{2 q}}\left(p_{k}(-y)^{q}\right)\right]+\right. \\
\left.+K_{\frac{1}{2 q}}\left(p_{k} \beta^{q}\right) J_{\frac{1}{2 q}-1}\left(p_{k}(-y)^{q}\right)\right]
\end{array}
$$

Из равенств (55) и (56) при любом $y \in[-\alpha, 0]$ следуют оценки:

$$
\begin{gathered}
\left|C_{k}^{\prime}(y)\right| \leqslant \frac{\widetilde{C}_{8} \sqrt{k} p_{k}(-y)^{q-1 / 2}}{I_{\frac{1}{2 q}}\left(p_{k} \beta^{q}\right) C_{0}} \mid J_{\frac{1}{2 q}}\left(p_{k} \alpha^{q}\right) J_{1-\frac{1}{2 q}}\left(p_{k}(-y)^{q}\right)+ \\
+J_{-\frac{1}{2 q}}\left(p_{k} \alpha^{q}\right) J_{\frac{1}{2 q}-1}\left(p_{k}(-y)^{q}\right) \mid \leqslant C_{10} k^{1+\lambda} e^{-k d} \\
\left|D_{k}^{\prime}(y)\right| \leqslant \frac{\widetilde{C}_{9} p_{k} \sqrt{k}(-y)^{q-1 / 2}}{C_{0}}\left[\left|J_{\frac{1}{2 q}-1}\left(p_{k}(-y)^{q}\right)-J_{1-\frac{1}{2 q}}\left(p_{k}(-y)^{q}\right)\right|+\right. \\
\left.+K_{\frac{1}{2 q}}\left(p_{k} \beta^{q}\right)\left|J_{\frac{1}{2 q}-1}\left(p_{k}(-y)^{q}\right)\right|\right] \leqslant C_{12} k^{1+\lambda}
\end{gathered}
$$

Поскольку $C_{k}^{\prime \prime}(y)=\left(p_{k} q\right)(-y)^{2 q-2} C_{k}(y)$ и $D_{k}^{\prime \prime}(y)=\left(p_{k} q\right)(-y)^{2 q-2} D_{k}(y)$, в силу оценок (53) и (54) при $-\alpha \leqslant y \leqslant-\varepsilon$ получим

$$
\left|C_{k}^{\prime \prime}(y)\right| \leqslant C_{13} k^{2}, \quad\left|D_{k}^{\prime \prime}(y)\right| \leqslant C_{14} k^{2} .
$$

ЛЕмма 3. При выполнении условия (39) при $k>k_{0}$ справедливы следующие оценки:

$$
\begin{gathered}
\left|u_{k}(y)\right| \leqslant C_{15}\left(\left|f_{k}\right|+\left|g_{k}\right|\right), \quad\left|v_{k}(y)\right| \leqslant C_{18}\left(\left|\widetilde{f}_{k}\right|+\left|\widetilde{g}_{k}\right|\right), \quad y \in[-\alpha, \beta] ; \\
\left|u_{k}^{\prime}(y)\right| \leqslant C_{16}\left(k\left|f_{k}\right|+k^{1+\lambda}\left|g_{k}\right|\right),\left|v_{k}^{\prime}(y)\right| \leqslant C_{19}\left(k\left|\widetilde{f}_{k}\right|+k^{1+\lambda}\left|\widetilde{g}_{k}\right|\right), y \in[-\alpha, \beta] ; \\
\left|u_{k}^{\prime \prime}(y)\right| \leqslant C_{17} k^{2}\left(\left|f_{k}\right|+\left|g_{k}\right|\right),\left|v_{k}^{\prime \prime}(y)\right| \leqslant C_{20} k^{2}\left(\left|\widetilde{f}_{k}\right|+\left|\widetilde{g}_{k}\right|\right), y \in[-\alpha, \varepsilon] \cup[\varepsilon, \beta] .
\end{gathered}
$$

Доказ ательств в. На основании формул (20), (29), (45) и (46) получим следующие представления для функций $u_{k}(y)$ и $v_{k}(y)$ :

$$
\begin{array}{ll}
u_{k}(y)=f_{k} A_{k}(y)+g_{k} B_{k}(y), & v_{k}(y)=\widetilde{f}_{k} A_{k}(y)+\widetilde{g}_{k} B_{k}(y), \quad y \geqslant 0, \\
u_{k}(y)=f_{k} C_{k}(y)+g_{k} D_{k}(y), & v_{k}(y)=\widetilde{f}_{k} C_{k}(y)+\widetilde{g}_{k} D_{k}(y), \quad y \leqslant 0 .
\end{array}
$$

Исходя из этих равенств на основании леммы 2 нетрудно получить указанные оценки.

Теперь на основании леммы 3 докажем, что функция $u(x, y)$, определяемая рядом (44), удовлетворяет условиям (2) и (3) поставленной задачи. Формально из ряда (44) почленным дифференцированием составим ряды:

$$
u_{x}=-\sqrt{\frac{2}{l}} \sum_{k=1}^{\infty} \lambda_{k} u_{k}(y) \sin \lambda_{k} x+\sqrt{\frac{2}{l}} \sum_{k=1}^{\infty} \lambda_{k} v_{k}(y) \cos \lambda_{k} x
$$




$$
\begin{gathered}
u_{y}=\frac{1}{\sqrt{l}} u_{0}^{\prime}(y)+\sqrt{\frac{2}{l}} \sum_{k=1}^{\infty} u_{k}^{\prime}(y) \cos \lambda_{k} x+\sqrt{\frac{2}{l}} \sum_{k=1}^{\infty} v_{k}^{\prime}(y) \sin \lambda_{k} x, \\
u_{x x}=-\sqrt{\frac{2}{l}} \sum_{k=1}^{\infty} \lambda_{k}^{2} u_{k}(y) \cos \lambda_{k} x-\sqrt{\frac{2}{l}} \sum_{k=1}^{\infty} \lambda_{k}^{2} v_{k}(y) \sin \lambda_{k} x \\
u_{y y}=\frac{1}{\sqrt{l}} u_{0}^{\prime \prime}(y)+\sqrt{\frac{2}{l}} \sum_{k=1}^{\infty} u_{k}^{\prime \prime}(y) \cos \lambda_{k} x+\sqrt{\frac{2}{l}} \sum_{k=1}^{\infty} v_{k}^{\prime \prime}(y) \sin \lambda_{k} x
\end{gathered}
$$

Тогда на основании леммы 3 ряды (44), (57), (58) при любых $(x, y) \in \bar{D}$ мажорируются числовым рядом

$$
C_{21} \sum_{k=1}^{\infty} k\left[\left|f_{k}\right|+\left|\widetilde{f}_{k}\right|+k^{1-\lambda}\left(\left|g_{k}\right|+\left|\widetilde{g_{k}}\right|\right)\right]
$$

а ряды $(59),(60)$ при любом $(x, y) \in \overline{D_{\varepsilon}}$ мажорируются рядом

$$
C_{22} \sum_{k=1}^{\infty} k^{2}\left(\left|f_{k}\right|+\left|\widetilde{f}_{k}\right|+\left|g_{k}\right|+\left|\widetilde{g_{k}}\right|\right)
$$

Лемма 4. Если функиии $f(x), g(x) \in C^{4}[0, l]$, на этом сегменте имеют кусочно-непрерывную производную третъего порядка и выполнены условия $f(0)=f(l), f^{\prime}(0)=f^{\prime}(l), g(0)=g(l), g^{\prime}(0)=g^{\prime}(l)$, то для коэфбфициентов $f_{k}$, $\widetilde{f}_{k}, g_{k}, \widetilde{g_{k}}$ справедливы следующие оценки:

$$
f_{k}=-\frac{p_{k}}{\lambda_{k}^{3}}, \quad \widetilde{f_{k}}=-\frac{\widetilde{p_{k}}}{\lambda_{k}^{3}}, \quad g_{k}=-\frac{s_{k}}{\lambda_{k}^{3}}, \quad \widetilde{g}_{k}=-\frac{\widetilde{s_{k}}}{\lambda_{k}^{3}},
$$

әде

$$
\begin{aligned}
& p_{k}=-\int_{0}^{l} f^{\prime \prime \prime}(x) \sin \lambda_{k} x d x, \quad \widetilde{p_{k}}=\int_{0}^{l} f^{\prime \prime \prime}(x) \cos \lambda_{k} x d x \\
& s_{k}=-\int_{0}^{l} g^{\prime \prime \prime}(x) \sin \lambda_{k} x d x, \quad \widetilde{s_{k}}=\int_{0}^{l} g^{\prime \prime \prime}(x) \cos \lambda_{k} x d x \\
& \sum_{k=1}^{+\infty} p_{k}{ }^{2}<+\infty, \quad \sum_{k=1}^{+\infty}{\widetilde{p_{k}}}^{2}<+\infty, \quad \sum_{k=1}^{+\infty} s_{k}{ }^{2}<+\infty, \quad \sum_{k=1}^{+\infty}{\widetilde{s_{k}}}^{2}<+\infty .
\end{aligned}
$$

Доказ а тель ст в о. Рассмотрим интегралы (12), (13), (27) и (28). Интегрируя их по частям три раза с учетом условий леммы, получим требуемые представления (63). Справедливость оценок (64) следует из неравенства Бесселя для тригонометрической системы.

В силу леммы 4 ряды (61) и (62) оцениваются соответственно числовыми рядами

$$
C_{23} \sum_{k=1}^{\infty}\left(\frac{\left|p_{k}\right|+\left|\widetilde{p_{k}}\right|}{k^{2}}+\frac{\left|s_{k}\right|+\left|\widetilde{s_{k}}\right|}{k^{1+\lambda}}\right)
$$


И

$$
C_{24} \sum_{k=1}^{\infty} \frac{1}{k}\left(\left|p_{k}\right|+\left|\widetilde{p_{k}}\right|+\left|s_{k}\right|+\left|\widetilde{s_{k}}\right|\right) .
$$

Поскольку числовые ряды (65), (66) сходятся, ряды (44), (57), (58) сходятся равномерно на $\bar{D}$, а ряды $(59),(60)$ - на $\overline{D_{\varepsilon}}$. Поэтому функция $u(x, y)$, определяемая равенством (44), удовлетворяет условиям (2) и (3).

Если для указанных в лемме 1 значений $\alpha_{q l}$ при $k=n \in\left\{n_{1}, n_{2}, \ldots, n_{m}\right\}$, где $1 \leqslant n_{1}<n_{2}<\cdots<n_{m} \leqslant k_{0}, n_{i}, i=\overline{1, m}$, и $m$-заданные натуральные числа, выполняется равенство $\delta_{n}(\alpha, \beta)=0$, то для разрешимости задачи (2)(6) необходимо и достаточно, чтобы выполнялись условия

$$
\begin{aligned}
& f_{n} \sqrt{\alpha} J_{\frac{1}{2 q}}\left(p_{k} \alpha^{q}\right)+g_{n} \sqrt{\beta} I_{\frac{1}{2 q}}\left(p_{k} \beta^{q}\right)=0, \quad n=\left\{n_{1}, n_{2}, \ldots, n_{m}\right\}, \\
& \widetilde{f}_{n} \sqrt{\alpha} J_{\frac{1}{2 q}}\left(p_{k} \alpha^{q}\right)+\widetilde{g}_{n} \sqrt{\beta} I_{\frac{1}{2 q}}\left(p_{k} \beta^{q}\right)=0, \quad n=\left\{n_{1}, n_{2}, \ldots, n_{m}\right\} .
\end{aligned}
$$

Тогда решение этой задачи определяется в виде суммы ряда

$$
\begin{array}{r}
u(x, y)=\frac{1}{\sqrt{l}} u_{0}(y)+ \\
+\left(\sum_{k=1}^{n_{1}-1}+\cdots+\sum_{k=n_{m-1}+1}^{n=n_{m}-1}+\sum_{k=n_{m}+1}^{+\infty}\right)\left[u_{k}(y) \cos \lambda_{k} x+v_{k}(y) \sin \lambda_{k} x\right]+ \\
+\sum_{n} u_{n}(x, y),
\end{array}
$$

где в последней сумме $n$ принимает значения $n_{1}, n_{2}, \ldots, n_{m}$ и функции $u_{n}(x, y)$ определяются по формуле

$$
\begin{gathered}
u_{n}(x, y)=\widetilde{u}_{n}(y) \cos \lambda_{k} x+\widetilde{v}_{n}(y) \sin \lambda_{k} x, \\
\widetilde{u}_{n}(y)= \begin{cases}\frac{1}{J_{\frac{1}{2 q}}\left(p_{k} \alpha^{q}\right)}\left[-g_{n} \alpha^{-\frac{1}{2}} \sqrt{y} I_{\frac{1}{2 q}}\left(p_{k} y^{q}\right)+b_{n} \sqrt{y} \delta_{n}(\alpha, y)\right], & y \geqslant 0, \\
\frac{1}{I_{\frac{1}{2 q}}\left(p_{k} \beta^{q}\right)}\left[-f_{n} \beta^{-\frac{1}{2}} \sqrt{-y} J_{\frac{1}{2 q}}\left(p_{k}(-y)^{q}\right)+b_{n} \sqrt{-y} \delta_{n}(-y, \beta)\right], & y \leqslant 0 ;\end{cases} \\
\widetilde{v}_{n}(y)= \begin{cases}\frac{1}{J_{\frac{1}{2 q}}\left(p_{k} \alpha^{q}\right)}\left[-\widetilde{g}_{n} \alpha^{-\frac{1}{2}} \sqrt{y} I_{\frac{1}{2 q}}\left(p_{k} y^{q}\right)+b_{n} \sqrt{y} \delta_{n}(\alpha, y)\right], & y \geqslant 0, \\
\frac{1}{I_{\frac{1}{2 q}}\left(p_{k} \beta^{q}\right)}\left[-\widetilde{f}_{n} \beta^{-\frac{1}{2}} \sqrt{-y} J_{\frac{1}{2 q}}\left(p_{k}(-y)^{q}\right)+b_{n} \sqrt{-y} \delta_{n}(-y, \beta)\right], & y \leqslant 0 .\end{cases}
\end{gathered}
$$

Конечные суммы в (69) следует считать нулями, когда верхний предел меньше нижнего.

Таким образом, доказана следующая

Теорема 2. Пусть числа $\alpha_{q l}$ удовлетворяют условиям леммы 1 , т.е. въполнена оценка (39) при всех $k>k_{0}$, и функции $f(x)$ u $g(x)$ удовлетворяют 
условиям леммы 4. Тогда если $\delta_{k}(\alpha, \beta) \neq 0$ при всех $k=\overline{1, k_{0}}$, то существует единственное решение задачи (2)-(6) и оно определяется рядом (44); если $\delta_{k}(\alpha, \beta)=0$ при $k=\overline{n_{1}, n_{m}} \leqslant k_{0}$, то задача (2)-(6) разрешима только тогда, когда выполнены условия (67) и (68) и решение в этом случае определяется рядом (69).

\section{3. Исследование задачи 2}

Пусть $u(x, y)$ - решение задачи (2)-(5). Рассуждая аналогично $\S 2$, введем функции (7)-(9). Относительно функции (7) получим краевую задачу (10)(12). Решение уравнения (1) ищется в классе (2), следовательно, для функций (14) должны выполняться условия сопряжения (11). Для этого найдем производные функций (14):

$$
u_{k}^{\prime}(y)=\left\{\begin{array}{cc}
a_{k} p_{k} y^{q-\frac{1}{2}} I_{\frac{1}{2 q}-1}\left(p_{k} y^{q}\right)-b_{k} p_{k} y^{q-\frac{1}{2}} K_{\frac{1}{2 q}-1}\left(p_{k} y^{q}\right), & y>0, \\
-c_{k} p_{k}(-y)^{q-\frac{1}{2}} J_{\frac{1}{2 q}-1}\left(p_{k}(-y)^{q}\right)- & \\
-d_{k} p_{k}(-y)^{q-\frac{1}{2}} Y_{\frac{1}{2 q}-1}\left(p_{k}(-y)^{q}\right), & y<0 .
\end{array}\right.
$$

В силу асимптотических формул для функций Бесселя в окрестности нуля (46) в случае, когда $1<s<2$, имеем особенности при $|y| \rightarrow 0$ вида

$$
\begin{gathered}
y^{q-\frac{1}{2}} K_{\frac{1}{2 q}-1}\left(p_{k} y^{q}\right)=O\left(y^{1-s}\right), \quad y>0, \\
(-y)^{q-\frac{1}{2}} Y_{\frac{1}{2 q}-1}\left(p_{k}(-y)^{q}\right)=O\left((-y)^{1-s}\right), \quad y<0 .
\end{gathered}
$$

Отсюда следует, что равенства (11) возможны только в том случае, когда $b_{k}=0$ и $d_{k}=0$ при всех $k \in \mathbb{N}$. Также для выполнения равенства (11) необходимо выполнение условия $c_{k}=-a_{k}$.

С учетом последних равенств решения (19) примут вид

$$
u_{k}(y)= \begin{cases}a_{k} \sqrt{y} I_{\frac{1}{2 q}}\left(p_{k} y^{q}\right), & y \geqslant 0 \\ -a_{k} \sqrt{-y} J_{\frac{1}{2 q}}\left(p_{k}(-y)^{q}\right), & y \leqslant 0 .\end{cases}
$$

Для нахождения постоянной $a_{k}$ в $(70)$ воспользуемся граничным условием (5) и формулой (12). Тогда получим

$$
a_{k}=\frac{f_{k}}{\sqrt{\beta} I_{\frac{1}{2 q}}\left(p_{k} \beta^{q}\right)} .
$$

Подставляя (71) в (70), найдем окончательный вид функции:

$$
u_{k}(y)= \begin{cases}\frac{f_{k} \sqrt{y}}{\sqrt{\beta} I_{\frac{1}{2 q}}\left(p_{k} \beta^{q}\right)} I_{\frac{1}{2 q}}\left(p_{k} y^{q}\right), & y \geqslant 0, \\ -\frac{f_{k} \sqrt{-y}}{\sqrt{\beta} I_{\frac{1}{2 q}}\left(p_{k} \beta q\right)} J_{\frac{1}{2 q}}\left(p_{k}(-y)^{q}\right), & y \leqslant 0 .\end{cases}
$$


Аналогично для функции (9) получаем краевую задачу (25)-(27). В этом случае единственное решение этой задачи определяется по формуле

$$
v_{k}(y)= \begin{cases}\frac{\tilde{f}_{k} \sqrt{y}}{\sqrt{\beta} I_{\frac{1}{2 q}}\left(p_{k} \beta^{q}\right)} I_{\frac{1}{2 q}}\left(p_{k} y^{q}\right), & y \geqslant 0, \\ -\frac{f_{k} \sqrt{-y}}{\sqrt{\beta} I_{\frac{1}{2 q}}\left(p_{k} \beta^{q}\right)} J_{\frac{1}{2 q}}\left(p_{k}(-y)^{q}\right), & y \leqslant 0 .\end{cases}
$$

Для нахождения функции (8) получим задачу (30)-(32), решение которой имеет вид

$$
u_{0}(y)=a_{0}(y-\beta)+f_{0},
$$

где $a_{0}-$ произвольная постоянная.

Исходя из формул (72)-(74) можно получить теорему о единственности решения задачи $(2)-(5)$, так как если $f(x) \equiv 0$ на $[0, l]$, то $u_{k}(y) \equiv 0, u_{0}(y) \equiv$ $\equiv a_{0}(y-\beta), v_{k}(y) \equiv 0$ для $k=1,2, \ldots$ на $[-\alpha, \beta]$. Тогда из $(7)-(9)$ при всех $y \in[-\alpha, \beta]$ и $k \in \mathbb{N}$ следуют равенства

$$
\begin{aligned}
& \int_{0}^{l} u(x, y) \cos \lambda_{k} x d x=0, \\
& \int_{0}^{l} u(x, y) d x=a_{0}(y-\beta), \\
& \int_{0}^{l} u(x, y) \sin \lambda_{k} x d x=0 .
\end{aligned}
$$

Введем функцию $v(x, y)=u(x, y)-a_{0}(y-\beta)$. Тогда равенства $(75)$ можно переписать в виде

$$
\begin{gathered}
\int_{0}^{l} v(x, y) \cos \lambda_{k} x d x=0, \\
\int_{0}^{l} v(x, y) d x=0, \\
\int_{0}^{l} v(x, y) \sin \lambda_{k} x d x=0 .
\end{gathered}
$$

Из равенств (76) в силу полноты системы (35) в пространстве $L_{2}[0, l]$ следует, что $v(x, y)=0$ почти всюду на $[0, l]$ при любом $y \in[-\alpha, \beta]$. Поскольку $v(x, y) \in C(\bar{D})$, имеем $v(x, y) \equiv 0$ или $u(x, y) \equiv a_{0}(y-\beta)$ на $\bar{D}$.

Следовательно, доказана следующая

Теорема 3. Если существует решение задачи (2)-(5), то оно единственно с точностью до слагаемого линейной бункиии по переменной $y: a_{0}(y-\beta)$.

Решение задачи (2)-(5) представляется в виде суммы ряда Фурье (44), где коэффициенты определяются формулами (72)-(74).

Лемма 5. Для функиий $u_{k}(y), v_{k}(y)$ и их производных при достаточно больших $k$ справедливы следующие оценки:

$$
\left|u_{k}(y)\right| \leqslant C_{31}\left|f_{k}\right|, \quad\left|v_{k}(y)\right| \leqslant C_{34}\left|\tilde{f}_{k}\right|, \quad y \in[-\alpha, \beta]
$$




$$
\begin{gathered}
\left|u_{k}^{\prime}(y)\right| \leqslant C_{32} k\left|f_{k}\right|, \quad\left|v_{k}^{\prime}(y)\right| \leqslant C_{35} k\left|\widetilde{f}_{k}\right|, \quad y \in[-\alpha, \beta] \\
\left|u_{k}^{\prime \prime}(y)\right| \leqslant C_{33} k^{2}\left|f_{k}\right|, \quad\left|v_{k}^{\prime \prime}(y)\right| \leqslant C_{36} k^{2}\left|\tilde{f}_{k}\right|, \quad y \in[-\alpha, \varepsilon] \cup[\varepsilon, \beta] .
\end{gathered}
$$

Доказ ат ельст в о проводится аналогично леммам 2 и 3.

Если функция $f(x)$ удовлетворяет условиям леммы 4, то ряд (44) и его производные до второго порядка включительно мажорируются сходящимися числовыми рядами с новыми коэффициентами (72)-(74).

Таким образом, приходим к следующему утверждению.

Теорема 4. Пусть $f(x) \in C^{3}[0, l]$ и выполнены условия $f(0)=f(l), f^{\prime}(0)=$ $=f^{\prime}(l), f^{\prime \prime}(0)=f^{\prime \prime}(l)$. Тогда задача $(2)-(5)$ разрешима и это решение определяется рядом (44) с точностью до слагаемого линейной функиии по переменной $y$, где коэфбициенты $u_{k}(y), v_{k}(y)$ и $u_{0}(y)$ определяются соответственно бормулами (72)-(74).

Конкурирующие интересы. Мы не имеем конкурирующих интересов.

Авторский вклад и ответственность. Все авторы принимали участие в разработке концепции статьи и в написании рукописи. Авторы несут полную ответственность за предоставление окончательной рукописи в печать. Окончательная версия рукописи была одобрена всеми авторами.

Финансирование. Исследование выполнялось без финансирования.

\section{Библиографический список}

1. Келдыш М. В. О некоторых случаях вырождения уравнений эллиптического типа на границе области // Докл. АН СССР, 1951. Т. 77, № 2. С. 181-184.

2. Кароль И. Л. Об одной краевой задаче для уравнения смешанного эллиптико-гиперболического типа // Докл. АН СССР, 1953. Т. 88, № 2. С. 197-200.

3. Франкль Ф. И. Обобщение задачи Трикоми и его применение к решению прямой задачи теории сопла Лаваля // Матем. сб., 1961. Т. 54(96), № 2. С. 225-236.

4. Сабитов К. Б. О единственности решения задачи Трикоми для одного уравнения смешанного типа второго рода / Дифференциальные уравнения с частными производными: Труды международной конференции, посвященной 70-летию академика С. Л. Соболева. Новосибирск: Наука, 1980. С. 48-50.

5. Сабитов К. Б. О постановке краевых задач для уравнения смешанного типа с вырождением второго порядка на границе бесконечной области // Сиб. матем. журн., 1980. Т. 21 , № 4. С. 146-150.

6. Сабитов К. Б. Задача типа Трикоми для уравнений смешанного типа с сильным характеристическим вырождением // Дифферени. уравнения, 1984. Т. 20, № 2. С. 333-337.

7. Франкль Ф. И. О задачах Чаплыгина для смешанных до- и сверхзвуковых течений // Изв. АН СССР. Сер. матем., 1945. Т. 9, № 2. С. 121-142.

8. Франкль Ф. И. Избранные труды по газовой динамике. М.: Наука, 1973. 703 с.

9. Шабат Б. В. Примеры решения задачи Дирихле для уравнений смешанного типа // Докл. АН СССР, 1957. Т. 112, № 3. С. 386-389.

10. Бицадзе А. В. Некорректность задачи Дирихле для уравнений смешанного типа в смешанных областях // Докл. АН СССР, 1958. Т. 122, № 2. С. 167-170.

11. Нахушев А. М. Критерий единственности задачи Дирихле для уравнения смешанного типа в цилиндрической области // Дифференц. уравнения, 1970. Т. 6, № 1. С. 190-191.

12. Солдатов А. П. Задача типа Дирихле для уравнения Лаврентьева-Бицадзе. І. Теоремы единственности // Докл. РАН, 1993. Т. 332, №6. С. 696-698.

13. Солдатов А. П. Задача типа Дирихле для уравнения Лаврентьева-Бицадзе. II. Теоремы существования // Докл. РАН, 1993. Т. 333, № 1. С. 16-18. 
14. Cannon J. R. A Dirichlet problem for an equation of mixed type with a discontinuous coefficient// Ann. Mat. Pura Appl., IV. Ser., 1963. vol.61. pp. 371-377. doi: 10.1007/ $\mathrm{BF} 02410656$.

15. Хачев М. М. Задача Дирихле для обобщенного уравнения Лаврентьева-Бицадзе в прямоугольной области // Дифферени. уравнения, 1978. Т. 14, № 1. С. 136-139.

16. Хачев М. М. Первая краевая задача для линейных уравнений смешанного типа. Нальчик: Эльбрус, 1998. 169 с.

17. Сохадзе Р. И. О первой краевой задаче для уравнения смешанного типа в прямоугольнике // Дифференц. уравнения, 1983. Т. 19, № 1. С. 127-134.

18. Сабитов К. Б. Задача Дирихле для уравнений смешанного типа в прямоугольной области // Докл. РАН, 2007. Т. 413, №1. С. 23-26.

19. Моисеев Е. И. О решении спектральным методом одной нелокальной краевой задачи // Дифферени. уравнения, 1999. Т. 35, № 8. С. 1094-1100.

20. Сабитов К. Б., Сулейманова А. Х. Задача Дирихле для уравнения смешанного типа второго рода в прямоугольной области // Изв. вузов. Матем., 2007. № 4. С. 45-53.

21. Сабитов К. Б., Сидоренко О. Г. Задача с условиями периодичности для вырождающегося уравнения смешанного типа // Дифферени. уравнения, 2010. Т. 46, № 1. С. 105-113.

22. Егорова И. П. Задача с условиями периодичности для уравнения смешанного типа с характеристическим вырождением // Вестн. СамГУ. Естественнонаучн. сер., 2009. № 8(74). С. 15-27.

23. Арнольд В. И. Малые знаменатели и проблемы устойчивости движения в классической и небесной механике // УМН, 1963. Т. 18, №6(114). С. 91-192.

24. Erdélyi A., Magnus W., Oberhettinger F., Tricomi F. G. Higher transcendental functions. vol. 2 / Bateman Manuscript Project. New York, Toronto, London: McGraw-Hill Book Co., Inc., 1953. xvii +396 pp. 


\title{
On the correctness of boundary value problems for the mixed type equation of the second kind
}

\author{
K. B. Sabitov ${ }^{1}$, I. P. Egorova ${ }^{2}$ \\ 1 Sterlitamak branch of the Bashkir State University, \\ Faculty of Mathematics and Information Technology, \\ 37, Lenina prospekt, Sterlitamak, 453103, Russian Federation. \\ 2 Samara State Technical University, \\ 244, Molodogvardeyskaya st., Samara, 443100, Russian Federation.
}

\begin{abstract}
In this paper, the intervals of change in the exponent of the degree of degeneration of a mixed-type equation with characteristic degeneration are established. The first boundary problem and the modified boundary problem (analogue of the Keldysh problem) with the conditions of periodicity are correctly set. In the case of the first problem, a criterion for the uniqueness of its solution is manifested. It is shown that the solution of the analogue of the Keldysh problem is unique up to a term of a linear function. Solutions are constructed as the sum of series of eigenfunctions of the corresponding one-dimensional spectral problem. In justifying the convergence of a series representing the solution of the first boundary-value problem, the problem of small denominators of a more complex structure arises in the class of regular solutions of this equation than in previously known works. The estimate on separation from zero is established with the corresponding asymptotic. Based on this estimate, sufficient conditions are found for the boundary functions to substantiate the uniform convergence of the series and their derivatives up to the second order inclusive.
\end{abstract}

Keywords: equations of mixed type, characteristic degeneration, boundaryvalue problems, periodicity conditions, spectral method, uniqueness, small denominators, existence.

Received: $1^{\text {st }}$ July, 2019 / Revised: $21^{\text {st }}$ August, 2019 /

Accepted: $16^{\text {th }}$ September, $2019 /$ First online: $11^{\text {th }}$ October, 2019

\section{Research Article}

(우(i) The content is published under the terms of the Creative Commons Attribution 4.0 International License (http://creativecommons.org/licenses/by/4.0/)

Please cite this article in press as:

Sabitov K. B., Egorova I. P. On the correctness of boundary value problems for the mixed type equation of the second kind, Vestn. Samar. Gos. Tekhn. Univ., Ser. Fiz.-Mat. Nauki [J. Samara State Tech. Univ., Ser. Phys. Math. Sci.], 2019, vol. 23, no. 3, pp. 430-451. doi: 10.14498/vsgtu1718 (In Russian).

\section{Authors' Details:}

Kamil B. Sabitov (1) https://orcid.org/0000-0001-9516-2704

Dr. Phys. \& Math. Sci., Professor; Head of Dept.; Dept. of Mathematical Analysis;

e-mail: sabitov_fmf@mail.ru

Irina P. Egorova (1D https://orcid.org/0000-0001-6023-1466

Cand. Phys. \& Math. Sci.; Associate Professor; Dept. of Higher Mathematics;

e-mail: ira. egorova81@yandex.ru 
Competing interests. We have no competing interests.

Authors' contributions and responsibilities. Each author has participated in the article concept development and in the manuscript writing. The authors are absolutely responsible for submitting the final manuscript in print. Each author has approved the final version of manuscript.

Funding. This research received no specific grant from any funding agency in the public, commercial, or not-for-profit sectors.

\section{References}

1. Keldysh M. V. On some cases of degenerate elliptic equations on the boundary of a domain, Dokl. Akad. Nauk SSSR, 1951, vol.77, no. 2, pp. 181-184 (In Russian).

2. Karol I. L. On a certain boundary value problem for an equation of mixed elliptic-parabolic type, Dokl. Akad. Nauk SSSR, 1953, vol. 88, no. 2, pp. 197-200 (In Russian).

3. Frankl F. I. A generalization of the Tricomi problem and its application to solution of the direct problem of the theory of the Laval nozzle, Mat. Sb. (N.S.), 1961, vol.54(96), no. 2, pp. 225-236 (In Russian).

4. Sabitov K. B. On the uniqueness of the solution of the Tricomi problem for a single equation of mixed type and second kind, In: Partial differential equations: differential equations and numerical mathematics, Work Collect. Conf.. Novosibirsk, 1980, pp. 48-50 (In Russian).

5. Sabitov K. B. Formulation of boundary-value problems for an equation of mixed type with degeneracy of second kind on the boundary of an infinite domain, Sib. Math. J., 1980, vol. 21, no. 4, pp. 591-594. doi: 10.1007/BF00995961.

6. Sabitov K. B. A problem of Tricomi type for equations of mixed type with strong characteristic degeneration, Differ. Uravn., 1984, vol. 20, no. 2, pp. 333-337 (In Russian).

7. Frankl F. I. On the problems of Chaplygin for mixed sub- und supersonic flows, Izv. Akad. Nauk SSSR, Ser. Mat., 1945, vol. 9, no. 2, pp. 121-142 (In Russian).

8. Frankl F. I. Izbrannye trudy po gazovoi dinamike [Selected Works on Gas Dynamics]. Moscow, Nauka, 1973, 703 pp. (In Russian)

9. Shabat B. V. Examples of solving the Dirichlet problem for equations of mixed type, Dokl. Akad. Nauk SSSR, 1957, vol. 112, no. 3, pp. 386-389 (In Russian).

10. Bitsadze A. V. Incorrectness of Dirichlet's problem for the mixed type of equations in mixed regions, Dokl. Akad. Nauk SSSR, 1958, vol. 122, no. 2, pp. 167-170 (In Russian).

11. Nakhushev A. M. An uniqueness condition of the Dirichlet problem for an equation of mixed type in a cylindrical domain, Differ. Uravn., 1970, vol. 6, no. 1, pp. 190-191 (In Russian).

12. Soldatov A. P. Dirichlet-type problems for the Lavrent'ev-Bitsadze equation. I. Uniqueness theorems, Dokl. Math., 1994, vol.48, no. 2, pp. 410-414.

13. Soldatov A. P. Dirichlet-type problems for the Lavrent'ev-Bitsadze equation. II. Existence theorems, Dokl. Math., 1994, vol.48, no. 3, pp. 433-437.

14. Cannon J. R. A Dirichlet problem for an equation of mixed type with a discontinuous coefficient, Ann. Mat. Pura Appl., IV. Ser., 1963, vol. 61, pp. 371-377. doi : 10.1007/BF02410656.

15. Hacev M. M. Dirichlet's problem for a generalized Lavrent'ev-Bitsadze equation in a rectangular region, Differ. Equ., 1978, vol. 14, no. 1, pp. 96-99.

16. Khachev M. M. Pervaia kraevaia zadacha dlia lineinykh uravnenii smeshannogo tipa [The First Boundary Value Problem for Linear Equations of Mixed Type]. Nalchik, Elbrus, 1998, 169 pp. (In Russian)

17. Sokhadze R. I. The first boundary value problem for an equation of mixed type in a rectangle, Differ. Uravn., 1983, vol. 19, no. 1, pp. 127-134 (In Russian).

18. Sabitov K. B. Dirichlet problem for mixed-type equations in a rectangular domain, Dokl. Math., 2007, vol. 75, no. 2, pp. 193-196. doi: 10.1134/S1064562407020056.

19. Moiseev E. I. On the solution of a nonlocal boundary value problem by the spectral method, Differ. Equ., 1999, vol. 35, no. 8, pp. 1105-1112. 
20. Sabitov K. B., Suleimanova A. Kh. The Dirichlet problem for a mixed-type equation of the second kind in a rectangular domain, Russian Math. (Iz. VUZ), 2007, vol.51, no.4, pp. 42-50. doi: 10.3103/S1066369x07040068.

21. Sabitov K. B., Sidorenko O. G. Problem with periodicity conditions for a degenerating equation of mixed type, Differ. Equ., 2010, vol.46, no.1, pp. 108-116. doi: 10.1134/ S0012266110010118.

22. Egorova I. P. The problem with periodicity conditions for the equations of mixed type with characteristic degeneracy, Vestnik Samarskogo Gosudarstvennogo Universiteta. EstestvennoNauchnaya Seriya, 2009, no. 8(74), pp. 15-27 (In Russian).

23. Arnol'd V. I. Small denominators and problems of stability of motion in classical and celestial mechanics, Russian Math. Surveys, 1963, vol.18, no. 6, pp. 85-191. doi: 10.1070/ RM1963v018n06ABEH001143.

24. Erdélyi A., Magnus W., Oberhettinger F., Tricomi F. G. Higher transcendental functions, vol. 2, Bateman Manuscript Project. New York, Toronto, London, McGraw-Hill Book Co., Inc., 1953, xvii+396 pp. 